\title{
Data-driven subtypes of major depressive disorder: a systematic review
}

\author{
Hanna M van Loo ${ }^{1}$, Peter de Jonge ${ }^{1 *}$, Jan-Willem Romeijn ${ }^{2}$, Ronald C Kessler ${ }^{3}$ and Robert A Schoevers ${ }^{1}$
}

\begin{abstract}
Background: According to current classification systems, patients with major depressive disorder (MDD) may have very different combinations of symptoms. This symptomatic diversity hinders the progress of research into the causal mechanisms and treatment allocation. Theoretically founded subtypes of depression such as atypical, psychotic, and melancholic depression have limited clinical applicability. Data-driven analyses of symptom dimensions or subtypes of depression are scarce. In this systematic review, we examine the evidence for the existence of data-driven symptomatic subtypes of depression.
\end{abstract}

Methods: We undertook a systematic literature search of MEDLINE, PsycINFO and Embase in May 2012. We included studies analyzing the depression criteria of the Diagnostic and Statistical Manual of Mental Disorders, fourth edition (DSM-IV) of adults with MDD in latent variable analyses.

Results: In total, 1176 articles were retrieved, of which 20 satisfied the inclusion criteria. These reports described a total of 34 latent variable analyses: 6 confirmatory factor analyses, 6 exploratory factor analyses, 12 principal component analyses, and 10 latent class analyses. The latent class techniques distinguished 2 to 5 classes, which mainly reflected subgroups with different overall severity: 62 of 71 significant differences on symptom level were congruent with a latent class solution reflecting severity. The latent class techniques did not consistently identify specific symptom clusters. Latent factor techniques mostly found a factor explaining the variance in the symptoms depressed mood and interest loss (11 of 13 analyses), often complemented by psychomotor retardation or fatigue (8 of 11 analyses). However, differences in found factors and classes were substantial.

Conclusions: The studies performed to date do not provide conclusive evidence for the existence of depressive symptom dimensions or symptomatic subtypes. The wide diversity of identified factors and classes might result either from the absence of patterns to be found, or from the theoretical and modeling choices preceding analysis.

Keywords: Major depressive disorder, subtypes, depressive symptoms, latent factor analyses, latent class analyses

\section{Background}

Major depressive disorder (MDD) is one of the most important challenges in global mental health $[1,2]$. In research, a continuing challenge is the diversity in the symptoms and pathophysiology of patients classified as having the disorder. MDD patients vary considerably in clinical presentation, course, treatment response, genetics and neurobiology [3-7]. One explanation for this diversity is that MDD has a polythetic definition; that is, a patient needs to satisfy some but not all symptoms. For the

\footnotetext{
* Correspondence: peter.de.jonge@umcg.nl

'Department of Psychiatry, University Medical Center Groningen, Hanzeplein

1, Groningen, $9713 \mathrm{GZ}$, The Netherlands

Full list of author information is available at the end of the article
}

diagnosis at least five of nine symptoms including at least one of the two core symptoms must be present [8]. It follows that there are 227 possible combinations of symptoms leading to this diagnosis. This is such a wide array of possibilities that two patients classified as having MDD might have only a single symptom in common. This diversity raises the question whether it makes sense for the purposes of comparisons in research to consider all the people who qualify for the diagnosis of MDD as having a single disorder.

To overcome the problem of symptom diversity, several attempts have been made to specify more homogenous subgroups within MDD. Subtypes have been proposed based on specific combinations of symptoms (for example,

\section{Biomed Central}


melancholic depression, psychotic depression), onset (seasonal affective disorder, postpartum, early versus late in life), course (single, recurrent, chronic), or severity [6]. Most subtyping schemes are based on pattern recognition and ordering using distinctions observed in clinical practice. For instance, 11 subtypes of MDD were proposed in the Research Diagnostic Criteria (RDC), the forerunner of the current Diagnostic and Statistical Manual of Mental Disorders (DSM), based on combinations of clinical characteristics, follow-up patterns, and findings from family studies [9-11]. However, the value of such distinctions has been called into question by the disappointing results of attempts to use these and subsequent subtyping distinctions in clinical practice $[6,12,13]$.

A different approach to discern useful subtypes with similar symptom profiles would be one that is data-driven; that is, which uses any of several statistical techniques to recognize patterns in reported symptoms of a heterogeneous group of subjects. These kinds of models have in common the fact that they reduce a large number of data from individuals to smaller numbers of latent variables based on similarity. Two dominant types of latent variable models are latent factor models and latent class models [14]. Latent factor models, such as exploratory factor analysis (EFA), reduce originally correlated variables to fewer latent factors (which might be specified as either correlated or uncorrelated) based on the correlations between the original variables $[15,16]$. By contrast, latent class models, such as cluster analysis (CA) and latent class analysis (LCA) cluster individuals rather than variables into relatively homogeneous subgroups. These subgroups are based on measures of similarity between each pair of individuals summed across all the variables considered in the analysis [17]. As both types of models are designed to discover structure in the absence of pre-existing hypotheses about subtypes, they provide useful approaches for examining heterogeneity based on distinctions that are not known beforehand [14].

Both latent factor and latent class models have been used to study the possible existence of useful MDD subtypes. However, findings in patients with MDD have not been systematically reviewed and thus the overall outcome is currently unclear. One of the questions, for instance, is whether a two-factor model applies to patients with MDD, as has been repeatedly found in patients with somatic illnesses. In somatically ill patients, latent factor analyses identified two main dimensions of depressive symptoms: one factor consisting of depressed mood, loss of interest, worthlessness, concentration problems and suicidality, and a second factor consisting of fatigue, appetite, sleep, and psychomotor disturbances $[18,19]$. The first set of symptoms is typically referred to as 'cognitive' or 'cognitive/affective', whereas the second set is typically referred to as 'somatic' or 'somatic/affective'; however, whether a cognitive and somatic symptom dimension is generalizable to patients with MDD or is limited to patients with somatic illnesses and comorbid depressive complaints is unknown. Systematic reviews or meta-analyses of studies empirically investigating the depressive symptom profiles of patients with MDD have not yet been performed.

In search of data-driven subtypes of MDD, we performed a systematic review of published studies that used these latent variable models to distinguish symptomatic subgroups or symptom dimensions for patients with MDD. Our main question was whether those studies identify consistent subtypes or symptom dimensions. Second, we evaluated whether the characteristic symptoms of empirically derived subtypes resemble the descriptions of current specifiers as melancholic and atypical [8]. Third, we studied whether latent factor analyses in patients with depression reveals a cognitive and somatic symptom dimension, as has been found in patients with somatic illnesses.

\section{Methods}

\section{Search strategy}

Studies were eligible for inclusion if they examined the existence of MDD subtypes by means of a latent variable analysis of depressive symptoms in patients with MDD. We searched three electronic databases MEDLINE (PubMed), Embase and PsycINFO for studies up to May 2012. We used the keywords 'major depressive disorder' and several synonyms for depressive subtype, symptom profile or symptom cluster (for full search strings, see Additional file 1). Many terms were used in the search, and were tested repeatedly for their success in finding relevant papers, based on our discovery of considerable variation in terminology across relevant articles. We used the database filters to exclude animal studies and studies with children. We did not use language restrictions. Retrieved articles were supplemented by studies cited in the reference lists of included studies plus a limited number of articles we found by hand searching.

\section{Inclusion criteria}

An article was eligible if it presented original data. As our primary interest was adult patients with MDD, we required that at least $75 \%$ of the studied subjects had to satisfy the criteria for MDD. We did not use a stricter criterion of $100 \%$ patients with MDD, because for reasons of completeness, we did not want to exclude studies with a minor percentage of patients with minor depression, adjustment disorder, or dysthymia. We included studies classifying patients using the criteria of the RDC, DSM (III or later versions), International Classification of Diseases (ICD; 9 or 10), and Geriatric Mental State-Automated Geriatric Examination for Computer Assisted 
Taxonomy (GMS-AGECAT) systems, based on the fact that the core symptoms of MDD in these different systems have a great deal of overlap [9,20-25]. To limit the diversity in the patient group, we excluded studies focusing on somatically ill patients with comorbid MDD. We selected all studies analyzing the existence of symptomatic subtypes by means of a latent variable statistical method. We were interested in all statistical methods capable of finding symptom dimensions or latent classes in the depressive symptoms. With regard to those symptoms, we organized results by the nine Criterion A symptoms of depression in the DSM-IV text revision, but with dichotomous distinctions made for the three compound psychophysiological symptoms in that diagnostic system (s3 (appetite/weight disturbance), s4 (sleep disturbance) and s5 (psychomotor disturbance)). These dichotomous distinctions (for example, between insomnia and hypersomnia) might have value in distinguishing subtypes [4] such as the previously suggested subtypes of 'typical' and 'atypical' depression [8]. Studies were included in our review if they performed a latent variable analysis on at least 6 of the 12 resulting disaggregated symptomatic criteria for MDD.

\section{Data extraction}

The database search described above resulted in 1,135 articles. Analysis of the reference lists of included articles provided another 29 relevant titles. Hand searching identified an additional 12 articles, giving a total of 1,176 unique titles. Subsequently, two independent raters $(\mathrm{HMvL}$ and PdJ reviewed the first half of articles, $\kappa=0.70$; HMvL and RAS reviewed the second half of articles, $\kappa=0.80$ ) reviewed the titles and abstracts of the identified studies to exclude any studies clearly not satisfying the inclusion criteria. If one (or both) of the reviewers assessed the title and abstract as possibly relevant, this article continued to be included in the review process. After this procedure, 93 studies remained included. We assessed the full text of all these 93 articles, and excluded 73 of them based on this review. In the end, 20 articles satisfied the inclusion criteria (see Additional file 2), containing 34 latent variable analyses in total (Figure 1).

\section{Data analysis}

The 34 analyses were examined as follows. First, we extracted the study characteristics from the papers, and in some cases obtained supplementary information from the authors. Second, we examined all the different questionnaires used in the measurement of depressive symptoms (which included the Hamilton Rating Scale for Depression (HRSD), the Beck Depression Inventory (BDI), the Montgomery and Åsberg Depression Rating Scale (MADRS), the Inventory for Depressive Symptomatology (IDS), and the Brief Psychiatric Rating Scale
(BPRS)). Using the original questionnaires, we determined which items of the different questionnaires corresponded to the 12 disaggregated depressive symptoms [26-34]. We then compared the results of the individual latent variable techniques. In the case of the latent factor techniques, we extracted the factors and the loadings of the 12 disaggregated symptoms on these factors. In the case of the latent class techniques, we extracted the reported latent classes and the differences in scores of the 12 symptoms in each class. The diversity in the questionnaires used precluded the performance of a mega-analysis or meta-analysis.

\section{Results}

\section{Literature search}

The 34 analyses in the 20 articles satisfying the inclusion criteria included 24 analyses that concerned the investigation of symptom dimensions by means of factor analyses $(\mathrm{n}=12)$ [35-39] or principal component analyses $(\mathrm{n}=12)$ [40-45], and 10 that concerned latent class analyses grouping a large number of individuals with depression into a smaller number of patient subgroups [42,45-53]. No analyses combined a latent factor approach with a LCA even though statistical techniques that allow this to be carried out do exist [14]. The total number of patients with depression was 7684. Samples included both men (38\%) and women $(62 \%)$, and both inpatients and outpatients. Overall, $96.6 \%$ of the study subjects satisfied the criteria for MDD. A small minority (3.4\%) of patients has been given other diagnoses such as minor depressive disorder or adjustment disorder, which were found in 7 of 20 articles (see Additional file 3, Table S1 and Table S2).

\section{Measurement of depressive symptoms}

In total, 11 different questionnaires (15 if we take into account the different versions of the HRSD, SCL, and IDS) were used to measure depressive symptoms in the 20 studies, whereas questionnaires measuring all 12 disaggregated symptoms were rare (3 of 11 , which were the IDS; the SADS, Schedule for Affective Disorders and Schizophrenia; and the Structured Clinical Interview for DSM (SCID)). In particular, few questionnaires measured both directions of the so-called somatic symptoms (s3 to s6). For instance, data on appetite/weight gain (s3b) and hypersomnia (s4b) were lacking in the majority of analyses. Overall, of the 34 latent variable analyses, only 6 analyses included all 12 disaggregated depressive symptoms. In general, substantially different questions were used to measure the same symptoms. These differences were especially marked for symptoms of feeling worthless or guilty (s7) and having diminished ability to think or concentrate, or increased indecisiveness (s8). Answer categories were ordinal in 15 studies, binary in 3 studies (yes/no dichotomized), and mixed in 2 studies. 


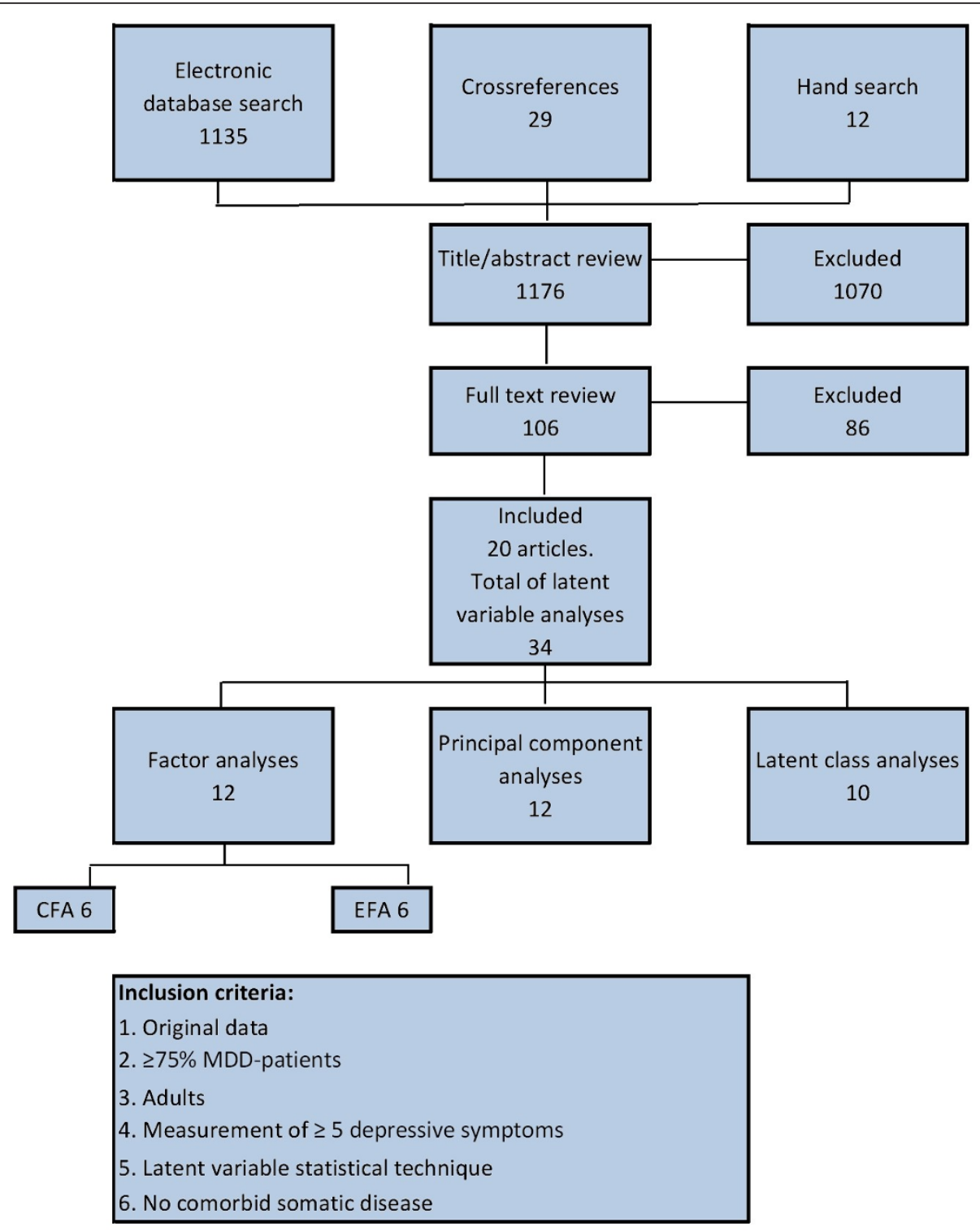

Figure 1 Flow chart of the review.

\section{Latent class analyses}

The 10 studies aimed at clustering patients based on symptom similarity comprised a total of 3,270 patients, with a mean of 327 patients analyzed per study (range 80 to 818 ). To visualize the effect of severity on class assignment, we sorted the original identified classes per study on overall severity. Therefore, we used the overall score of the class on each complete questionnaire, with class (a) being the most severe class of the study, class (b) being less severe than (a) and so on (see Additional file 3, Table S3). In total, there were 71 significant differences in symptom scores between classes. Notably, 62 of the 71 significant differences were related to class severity; that is, the overall most severe class scored higher on the symptom than the overall less severe class (Figure 2). This is in line with the idea that these statistical techniques are specifically able to separate more severe classes from less severe classes [14]. This pattern was especially clear in four of these studies [45-48]. Only nine significant differences deviated from this result $[49,53]$ (Figure 3); all nine of these exceptions concerned so-called somatic depressive symptoms, and were present in latent class analyses of the two largest samples of patients $(\mathrm{n}=569$ and $\mathrm{n}=818)$, in which patients in a less severe class scored higher than patients in a more severe class for fatigue (s6), weight (s3), and sleep disturbances (s4). 


\begin{tabular}{|c|c|c|c|c|c|c|c|c|c|c|c|}
\hline \multicolumn{2}{|c|}{ Depressive symptoms } & \multirow{2}{*}{\begin{tabular}{|l|} 
Andreasen \\
$b, a>c$ \\
\end{tabular}} & \multirow{2}{*}{\begin{tabular}{|l|} 
Maes \\
$\mathrm{a}, \mathrm{b}$ \\
\end{tabular}} & \multirow{2}{*}{\begin{tabular}{|l|} 
Schotte \\
$a>b$
\end{tabular}} & \multirow{2}{*}{$\begin{array}{l}\text { Hybels } \\
a, b, c, d\end{array}$} & \multirow{2}{*}{\begin{tabular}{|l|} 
Lamers \\
$a>b>c$ \\
\end{tabular}} & \multirow{2}{*}{$\begin{array}{l}\text { Parker } \\
\end{array}$} & \multirow{2}{*}{\begin{tabular}{|l} 
Davidson 88 \\
$a=b=c, d=e$
\end{tabular}} & \multirow{2}{*}{$\begin{array}{l}\text { Davidson } 89 \\
\mathrm{a}=\mathrm{c}, \mathrm{d}, \mathrm{b}, \mathrm{e}\end{array}$} & \multirow{2}{*}{$\begin{array}{l}\text { Grove } \\
a, b>c\end{array}$} & \multirow{2}{*}{\begin{tabular}{|l|} 
Haslam \\
$a, c, b, d$
\end{tabular}} \\
\hline 1 & depressed mood & & & & & & & & & & \\
\hline 2 & anhedonia & $b, a>c$ & $a, b$ & $a>b$ & $a, b, c, d$ & $a, b>c$ & $b, a>c$ & $a=b=c, d=e$ & $b, e, a, c, d$ & $b, a>c$ & $a, d, b, c$ \\
\hline $3 a$ & weight loss & $b, a>a, c$ & $a, b$ & $a>b$ & $a, b, c=d$ & $b>a>c^{*}$ & $a, b>c$ & $a, b, c, d, e$ & $c, a, e, b, d$ & $b>a>c$ & $a, b, c, d$ \\
\hline $3 \mathrm{~b}$ & weight gain & $b, c, a$ & - & - & - & $b>a>c^{*}$ & - & d,e,b,c,a & - & $a>c>b$ & - \\
\hline $4 a$ & insomnia & $b, a>c$ & $a, b^{*}$ & $a>b^{*}$ & $a, b, c=d$ & $b>a>c^{*}$ & $a, b, c$ & $\cdots, e \#$ &..,$b$, e\# & $b>a>c$ & $a, b, c, d$ \\
\hline $4 \mathrm{~b}$ & hypersomnia & $c, b, a$ & $a, b^{*}$ & $a>b^{*}$ & - & $b>a>c^{*}$ & - & $c, e, a, d=b$ & - & - & - \\
\hline $5 a$ & psychomotor agitation & $a, b>c$ & $a>b^{*}$ & $a>b^{*}$ & - & $a>b>c^{*}$ & $a, b>c$ & $a, b, d, c, e$ & $c, e, a, b, d$ & $b, a>c$ & - \\
\hline $5 \mathrm{~b}$ & psychomotor retardation & $a, b, c$ & $a>b^{*}$ & $a>b^{*}$ & $a, b, c, d$ & $a>b>c^{*}$ & $a, b>c$ & $c, a, b, d, e$ & $a, b, d, e, c$ & - & - \\
\hline 6 & fatigue & $a, b>b, c$ & $a>b$ & $a>b$ & - & $b>a>c$ & - & $a, b, e, c, d$ & $a, b, d, e, c$ & $b, a>c$ & $b, a, c, d$ \\
\hline 7 & worthlessness & $a>b, c$ & $a, b$ & $a>b$ & $a, b, c, d$ & $b, a>c$ & $a>c, b$ & $c, b, a, d, e$ & $c, b, a, d, e$ & $b, a>c$ & $c, a, b, d$ \\
\hline 8 & ability to think & $a, b>c$ & $a>b$ & $a>b$ & $a, b, c=d$ & $b, a>c$ & - & - & $c, a, b, d, e$ & $b, a>c$ & $a, b, c, d$ \\
\hline 9 & suicidality & $a, b>c$ & $a, b$ & $a>b$ & $a, b, c, d$ & $a>b>c$ & . & $b, c, a, e, d$ & $c, a, b, e, d$ & $a, b>c$ & $c, a, b, d$ \\
\hline
\end{tabular}

\section{key to symbols}

$\mathrm{a}>\mathrm{b}$ cluster a scores significantly higher than cluster b on symptom

$\mathrm{a}<\mathrm{b}$ cluster a scores significantly lower than cluster $\mathrm{b}$ on symptom

a , b cluster a scores higher than cluster b on symptom, not significant

* measured by 1 compound symptom (e.g. sleep disorders instead of insomnia versus hypersomnia) not recorded

exactly same score

a class a scores significantly different on sx compared to mean score on symptom

significant difference with overall more severe class $>$ less severe class ( $>>b, a>c$ or $b>c)$

significant difference with overall more severe class $<$ less severe class (e.g. a $<$ b, a $<$ c, b $<$ c)

\# sequence $a \cdot d$ is dependent on early/middle/late insomnia

Figure 2 Latent class analyses

\section{Latent factor analyses}

Our search yielded 24 analyses aimed at discovering dimensions of depressive symptoms. In total, there were 12 factor analyses and 12 principal component analyses (PCA) from 12 studies (some studies performed different analyses on the same patient sample). The factor analysis studies concerned six exploratory factor analyses (EFA) and six confirmatory factor analyses (CFA). The 12 factor analyses were performed on larger studies, with a mean of 562 patients per study (range 96 to 1049 , total $n=3369$ ). The 12 PCA concerned on average 174 patients (range 60 to 400 , total $\mathrm{n}=1045$ ).

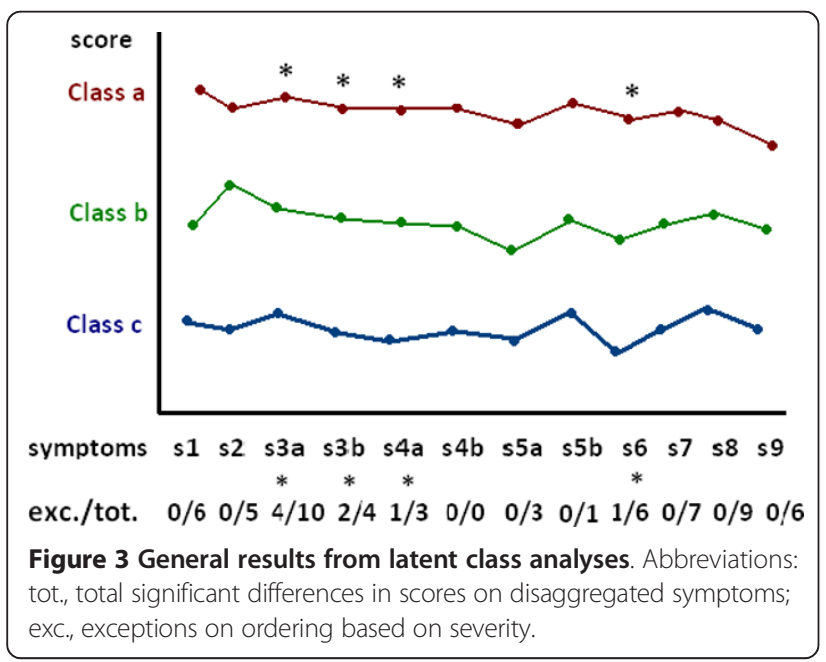

\section{Confirmatory factor analyses}

In two studies, CFA was used to test how well a single factor explained the variance in depressive symptoms $[35,36]$ (Table 1). The measures for model fit (Confirmatory Fit Index, Tucker-Lewis Index, and Root Mean Square Error of Approximation) showed that a single factor explained about $50 \%$ of variance in depressive symptoms.

\section{Exploratory factor and principal component analyses}

Nine studies exploratorively analyzed the dimensions underlying items of single questionnaires by means of PCA and EFA. The resulting factors of the 10 PCA and 3 EFA differed considerably (Figure 4). First, the number of derived factors varied from 2 to 7 (mean 3.5) explaining between $36.8 \%$ and $79.3 \%$ of total variance (mean $55 \%$ ). Second, the item content of the identified factors varied substantially. Concerning the cognitive symptoms (s1, s2, s7, s8, s9) of patients with depression, 10 of 13 analyses included all 5 cognitive symptoms, but of those 10, there was not a single study in which all 5 cognitive symptoms loaded on the same factor. In 3 of the 10 studies, 4 of 5 cognitive symptoms loaded on the same factor $[39,41,45]$, and in another 4 studies, 3 of the 5 cognitive symptoms loaded on the same factor $[38,43,44]$. Only one study had a factor on which four cognitive symptoms and no somatic symptoms loaded [39]; at least one somatic symptom loaded on the remaining six factors with three or more cognitive symptoms.

With regard to the somatic symptoms (s3 to s6), 8 of 13 studies measured the 4 somatic symptoms of depression, 
Table 1 Confirmatory factor analyses.

\begin{tabular}{llllllll}
\hline Author & Number of factors & Items in analysis & Explained variance & TLI & CFI & RMSEA & Factor correlation \\
\hline Uher [12] & 1 & MADRS & 0.57 & 0.99 & 0.97 & 0.10 & NA \\
& 1 & HRSD-17 & 0.36 & 0.93 & 0.87 & 0.09 & NA \\
& 1 & HRSD-6* & 0.48 & 0.99 & 0.98 & 0.07 & NA \\
& 1 & BDI & 0.48 & 0.97 & 0.88 & 0.10 & NA \\
& 1 & All 48 items & 0.45 & 0.90 & 0.63 & 0.17 & NA \\
Lux [35] & 1 & 9 MDD symptoms & - & 0.97 & 0.96 & 0.07 & NA \\
& 2 & 9 MDD symptoms & - & 0.97 & 0.97 & 0.06 & 0.83 \\
\hline
\end{tabular}

Abbreviations: BDI, Beck Depression Inventory; CFI, Confirmatory Fit Index; HRSD, Hamilton Rating Scale for Depression; MADRS, Montgomery and Åsberg Depression Rating Scale; MDD, major depressive disorder; NA, not applicable; * HRSD-6 contains 6 items out of the HRSD-17 (items 1,2,7,8,10,13) corresponding to depressive symptoms $1,2,5 \mathrm{~b}, 6,7 ;$ - not recorded.

with at least one of the disaggregated symptoms of appetite/weight, sleep, and psychomotor disturbance included, and none of these 8 studies had a common factor on which all 4 , or 3 of the 4 , somatic symptoms loaded. In 7 out of 8 analyses, at least one factor was identified that explained the variance of 2 of the 4 somatic symptoms [39-41,44,45], and the total number of factors with 2 somatic symptoms was 10, describing the variance of 6

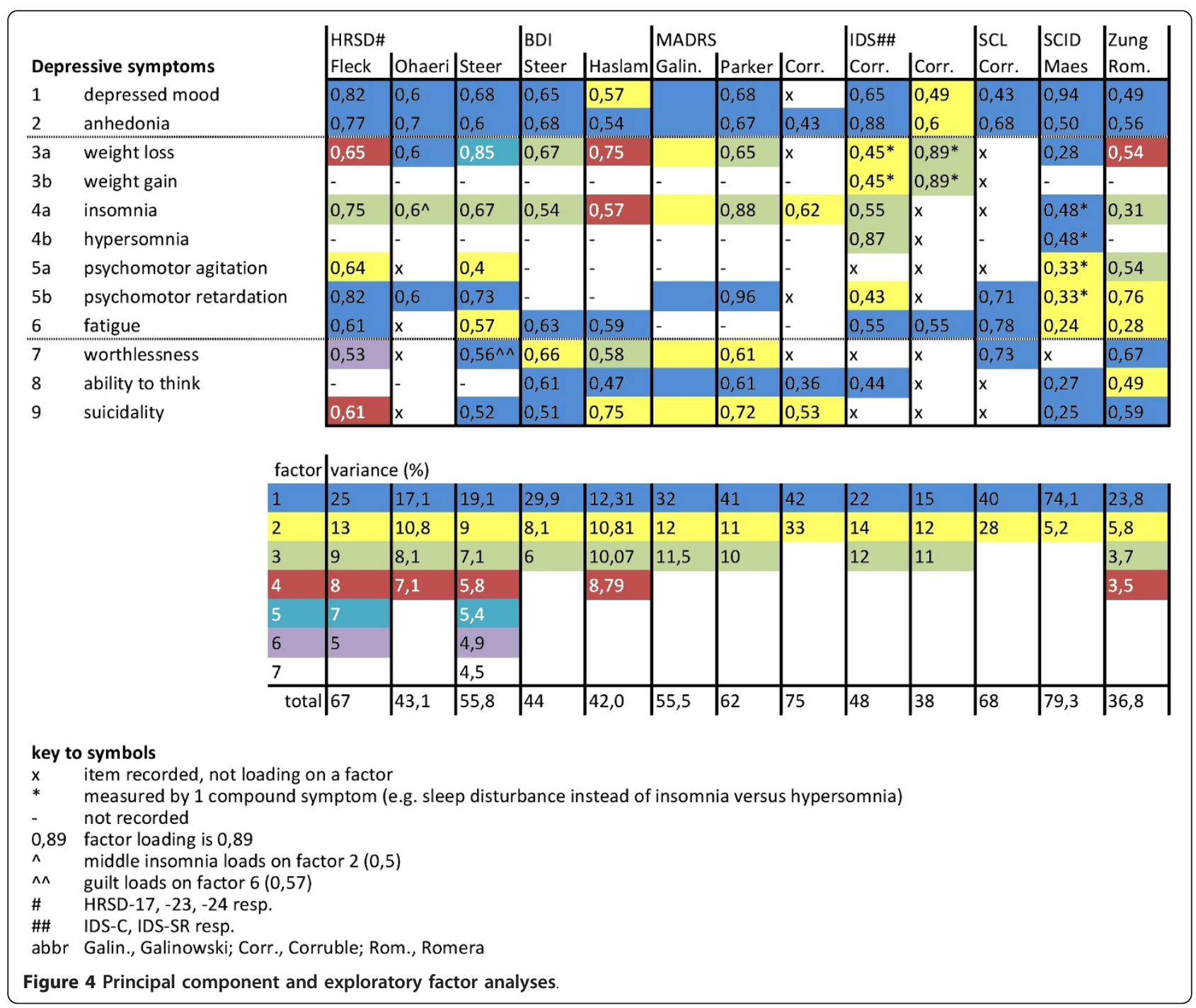


different pairs of somatic symptoms. Of those 10 factors with 2 somatic symptoms, 5 also incorporated one or more cognitive symptoms $[37,39,40,44,45]$.

The most consistent finding was that the 2 core symptoms of depression (sadness and loss of interest; s1 and s2), loaded on one single factor in 11 out of 13 analyses [37-41,43-45]. In 9 of these 11 analyses, s1 and s2 loaded on factor 1, which was the factor explaining most of the variance. Psychomotor retardation and/or fatigue ( $55 \mathrm{~b}$ and s6) also loaded on the same factor in 8 of 11 analyses [38,40-44]. Thus, the most prevalent finding was one single factor explaining the variance of a mixture of cognitive and somatic symptoms (s1, s2, s5b, and s6).

Figure 4 shows the diversity in the retrieved factors, such as the number, the item content, and loadings. Figure 5 was derived from Figure 4, and shows the proportion of pairwise common factor loadings. In total, the studies described 461 measurements of pairs of symptoms (for example, the combination of s1 and s2 was measured in all 13 studies, while the combination of $\mathrm{s} 1$ and s5a was measured in 7 studies). Of these 461 measurements of symptom pairs, 97 pairs loaded on a common factor, resulting in an average proportion of pairwise common factor loadings of 0.21 (97/461). In Figure 5, the heights of the 45 vertical bars depend on the proportion of each pair of symptoms that loaded on a common factor; for example, pair s1 and s2 loaded 11 out of 13 times on a common factor, resulting in a proportion of 0.85 pairwise common factor loadings. In case of a clear two-factor solution, one would expect to find a landscape of two areas with high bars, and one area with low bars. Obviously this is not the case, and neither can those areas be found by rearranging the symptom orders on the $\mathrm{x}$ - or $\mathrm{z}$-axis. Furthermore, for relatively many symptom pairs the proportion of common factor loadings approached the average of 0.21 , which is not consistent with a clear two-factor solution. Of note, weight gain (s3b) and hypersomnia (s4b) were measured too infrequently to include in this figure.

Figure 6 was also derived from Figure 4, and shows all depressive symptom combinations that were found to load on a common factor. In total, 27 factors had an item content of 2 or more depressive symptoms. The item content of those 27 factors was unique in 22 factors; only 5 factors had a non-unique item content, with two symptom combinations occurring more than once (Figure 6).

\section{Influence of different patient samples (same variables)}

Several studies analyzed the same questionnaires with different patient samples: three studies used the HRSD $[37,40,41]$, two studies the BDI $[41,42]$ and three studies the MADRS $[38,43,44]$. The analyses of the same questionnaires resulted in substantial different factors, both qualitatively (item content) and quantitatively (number

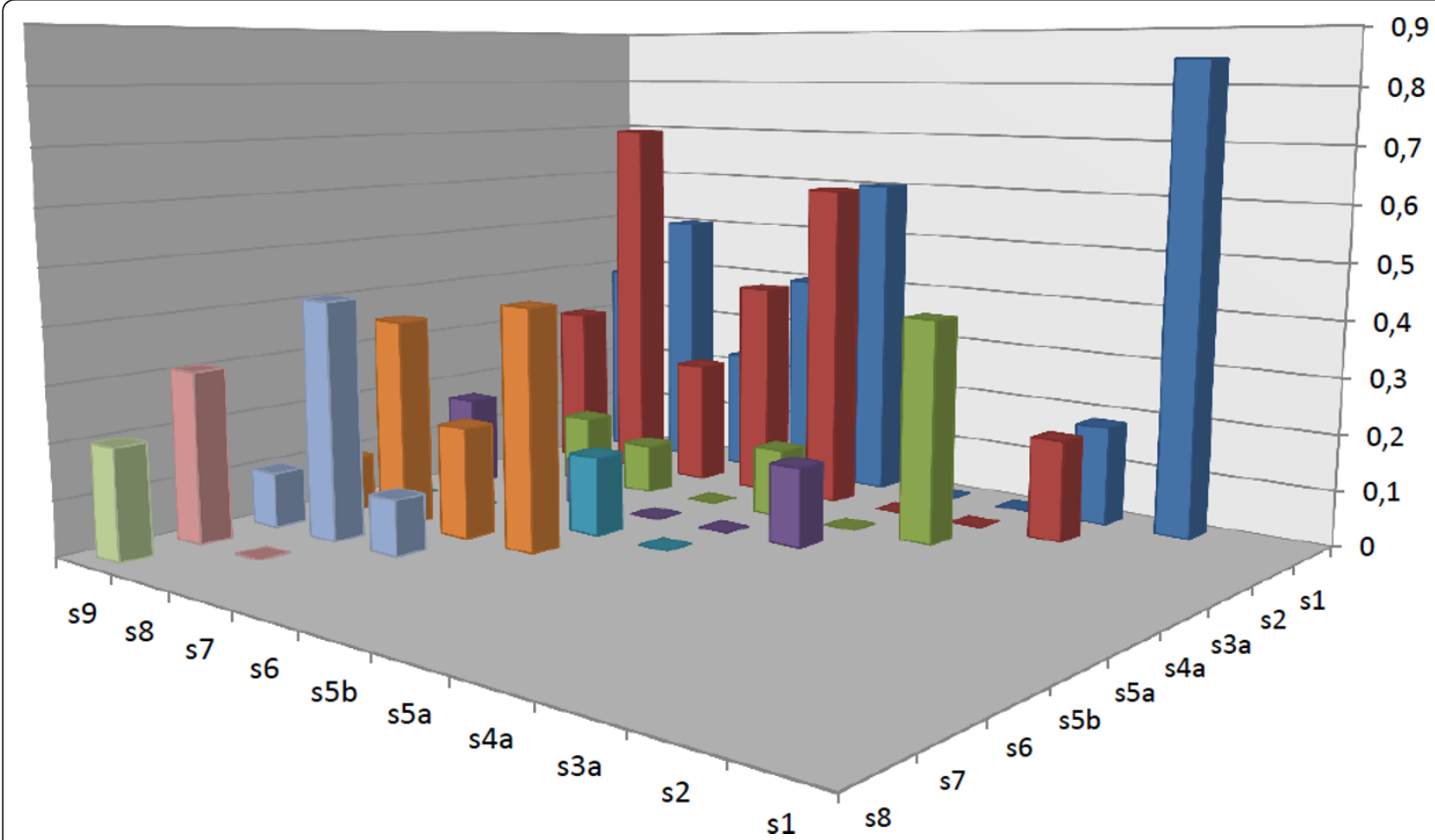

Figure 5 Pairwise common factor loadings. The $x$ - and $z$-axes show disaggregated depressive symptoms, while the $y$-axis shows the proportion of pairwise common factor loadings. 


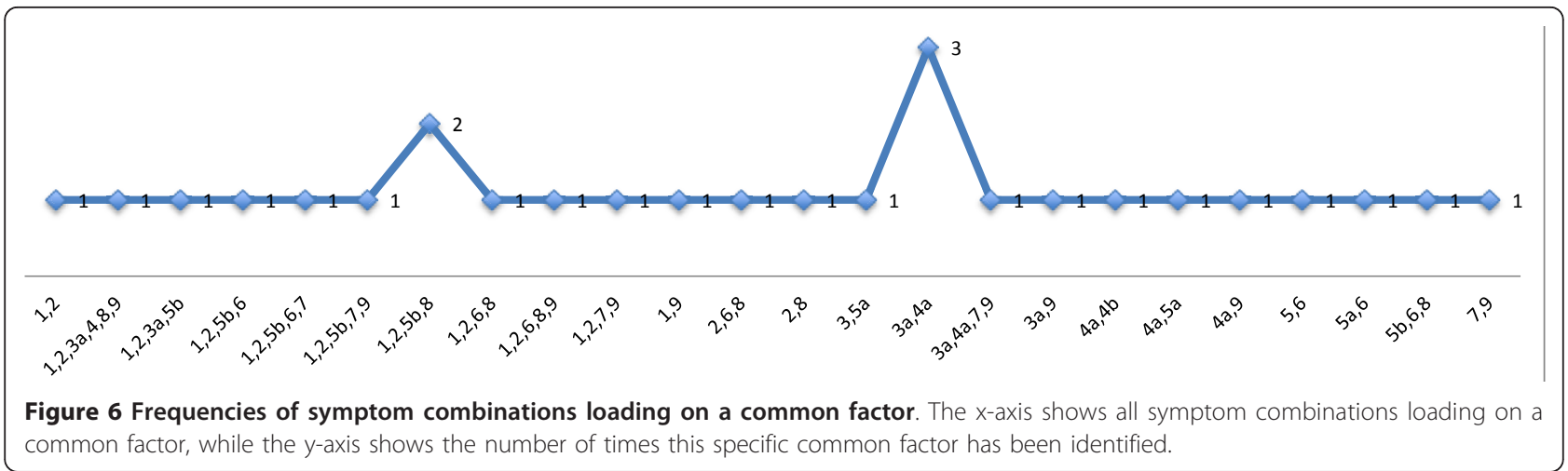

of factors). Varying influences of the patient sample on the resulting dimensional structure can be seen from the collected analyses (Figure 4). First, differences in severity affected the factorial structure. Galinowski and Lehert performed two PCA of the MADRS from the same group of patients at a different timepoint [43]. On day 0, before antidepressant treatment, three components explained $55.5 \%$ of the variance of the MADRS items, whereas after 28 days of antidepressant treatment, one component explained $66 \%$ of the variance. A likely explanation is the diminished overall severity score on the MADRS (from 35 to 17 points), with a decrease in correlations between items. However, such severity differences do not explain all variety in factor structure. This is illustrated by the studies of Corruble et al. and of Galinowski and Lehert $[43,44]$, which both had a patient sample with a roughly similar mean MADRS score ( 34 vs. 35 points), yet, the principal components derived from the samples differ considerably (two versus three PCs, explaining $75 \%$ vs. $55.5 \%$ of the variance with a different item content). Similar diverging factor patterns resulted from the studies analyzing the BDI or HRSD.

\section{Influence of different questionnaires (same patient sample)}

Two studies investigated the factor structure of different questionnaires from the same patient sample in separate analyses. First, Steer et al. performed two PCA on the BDI and the HRSD, which resulted in three and seven qualitatively different components, respectively [41](Figure 4). Second, Corruble et al. performed four PCA on the IDS-C, IDS-SR, MADRS and the SCL-90R [44], and the variety in the resulting factor structure was again substantial. Even the two PCA on the IDS Clinician (IDS-C) and IDS SelfReport (IDS-SR) ratings produced components with a different item content, though the questionnaires are largely similar.

\section{Combining items of multiple questionnaires in one analysis}

Four studies performed latent factor analyses on a combination of items from different questionnaires $[36,37,41,54]$. Although response categories vary between different questionnaires, no study used a smoothing method to adjust for these variances, which could explain part of the following results. Steer et al. performed PCA on a combination of all HRSD and BDI items. All BDI items loaded on principal component 1 (PC1), whereas the HRSD items loaded on PC2 and PC3 [41]. A largely similar result was found by Uher et al., using EFA on 47 items from the MADRS, the HRSD, and the BDI. The depressive symptom items of the MADRS and the HRSD predominantly loaded on factor 1 , whereas BDI items mainly loaded on factor 3 [36]. Thus, those studies show a high correlation of items in one questionnaire. The opposite result was found by Gullion and Rush [54], who performed EFA on 88 items of the IDS-C, IDS-SR, HRSD, and BDI. They identified 10 factors mainly explaining the variance of items measuring the same symptom in the different questionnaires; for example, factor 2 comprised several items measuring guilt/worthlessness (s7), for instance 'guilt feelings' (BDI), 'guilt feelings' (HRSD), 'self-criticism and blame' (IDS-SR, IDS-C).

Ohaeri and Otote performed the fourth analysis in which items of different questionnaires are included, that is, items of the HRSD and the BPRS [37]. Of note, these authors also performed EFA on the HRSD items alone (Figure 4), therefore, the effect of the extra BPRS items on the factorial structure is directly observable in this study. First, EFA of the HRSD items exclusively resulted in four factors, explaining $43.1 \%$ of the variance. The first factor comprised six items: depressed mood, anhedonia, weight and appetite loss, psychomotor retardation, and paranoid symptoms, and this factor is described as 'core depressive'. Second, an EFA on the HRSD plus BPRS items also resulted in four factors, explaining $43.7 \%$ of the variance. The first factor is again described as 'core depressive'; however, this factor now contains different items. Of the previous six HRSD items only two remained: depressed mood and psychomotor retardation. By contrast, the item with the highest factor loading was uncooperativeness (0.8). In addition, the factor contained hypochondriasis, insight loss, and emotional withdrawal. A comparable shift in item content was found for the other factors. 


\section{Discussion}

Our results show that studies performed to date provide insufficient evidence to confirm the stable existence of clear data-driven symptomatic subtypes of depression. First, relatively few studies have been dedicated to the detection of data-driven MDD subtypes (20 of 1176 articles). Second, the outcomes of these few studies are conflicting. Latent class analyses mainly grouped patients on overall severity, but not in classes with qualitatively different symptom profiles, whereas latent factor analyses, most consistently identified a factor explaining the variance of a mixture of cognitive and somatic symptoms (s1, s2, s5b, s6), which seems in contradiction with a purely cognitive or somatic symptom dimension. However, the 13 identified factors differed to such an extent that generalizable conclusions are questionable. In short, the collected studies fail to give adequate evidence for the existence of qualitatively different subtypes or symptom dimensions of MDD. Thus, this lack of empirical support also holds for theoretical motivated subtypes such as as melancholic or atypical, or cognitive and somatic symptom dimensions in MDD.

Particularly notable is the great deal of diversity in the results. How can we explain this large diversity? From the collected results, it can be seen that all sorts of factors influence the outcomes of latent variable analyses; for instance, the included number of patients, and the severity and quantity of their symptoms obviously affect the resulting latent classes and factors. Presumably, there is considerable difference in symptom endorsement rates between the studies, as was reported from a recent mega-analysis of genome-wide association studies of patients with MDD [55]. Furthermore, the extensive number and differences of the questionnaires used, including all not recorded symptoms (weight gain, hypersomnia), is a likely contributor to the diverging results. On top of that, previous studies have found that in case of latent factor analyses, several items, including model choice (for example, component or common factor model), sample size, number and communalities of the variables, selection of the number of factors and model fit criteria, rotation method, and the degree of overdetermination, affect the stability and correspondence of the resulting factors [56-58]. An extra influence to take into account is the manifestly high correlation of symptoms within one questionnaire, as shown by some (not all) latent factor analyses on different questionnaires simultaneously. Thus, many theoretical choices preceding analysis are important determinants of the retrieved classes or dimensions.

The major insight from this review is that we should improve our research techniques considerably to find data-driven subtypes of depression. Of course, it is an open-ended question as to whether such a pattern exists.
It is possible that there simply are no symptomatic subtypes or dimensions, and that latent variable techniques have failed to show consistent subtypes because of this fact. One could argue that if there were clear symptomatic subtypes or dimensions, a more consistent pattern would have emerged out of the data, regardless of the theoretical and measurement choices involved. The other possibility is that symptomatic subtypes and dimensions do exist, but that the techniques used to date did not succeed in identifying them. If the second possibility is true, a crucial question is how study methods could be improved to detect patterns in the data that have not yet been detected. Careful consideration of all theoretical and modeling choices that influence study outcomes will be required to answer that question.

One possible strategy to improve study methods is data enrichment, because it is clear that the quality of data crucially determines the quality of study outcomes. First, some of the studies reviewed above indicate the possible benefit of dynamic measurements, as they showed that differentiated symptom profiles might be clearer with more rather than less severe cases or for patients earlier rather than later in the treatment process. Therefore it would seem prudent to study changes in symptom structure and severity over the course of treatment, preferably also distinguishing the influence of medication. A second set of choices would involve the scales used to assess these symptoms. Some diagnostic instruments use dichotomous (yes/no) measures for each symptom, whereas others have gradual assessments. Gradual assessments, would of course be expected to provide textured differentiation, and in this way possibly lead to greater precision in detecting meaningful subtypes. Another concern is that the frequently used rating scales are primarily designed to be sensitive to change as opposed to capturing the detailed phenomenological picture of MDD. To address the potential heterogeneity in patients with depression, at least all DSM criteria, including all disaggregated symptoms (s3s5), should be measured in a standardized fashion. A third possibility to enrich data would be the inclusion of other variables in addition to depressive symptoms in analyses. Concerning the symptoms to include in the evaluation, it seems clear that this set of symptoms should be a broad one, so as to allow for the possibility of detecting subtypes associated with symptoms beyond those in the current DSM and ICD systems. For example, there is some suggestion in the literature that there might be value in differentiating irritable from non-irritable MDD [59] and in assessing the influence of anxiety [60,61]. Moreover, apart from symptoms, other indicators, such as hormone status, genetic profile, sex, and age, could also be included as variables in future analyses. The inclusion of these non-symptomatic variables possibly contributes to the 
unraveling of causal mechanisms of depressive subtypes, although we firmly believe that symptoms are the basis on which to start. Symptoms are non-invasive measures of disease; clinicians are trained to recognize and classify patients based on symptoms; and data on symptoms have been collected world-wide.

A second strategy to improve study methods involves the statistical approaches used to uncover depressive dimensions and subtypes. In addition to the latent factor and latent class approaches, we should consider complex CA models, especially those that use a canonical formulation to predict diverse outcomes [62,63], and mixture models that combine features of latent class and item response theory models [64] or latent class and latent factor models [14]. Some recent studies have used factor mixture analyses to identify subtypes in other psychiatric disorders, such as attention-deficit/hyperactivity disorder [65], post-traumatic stress disorder [13], and schizophrenia [66]. To date, these approaches have not yet been used to search for subtypes of MDD, but are attractive alternatives in light of their success in detecting useful subtypes of other disorders. Obviously, in accordance with the described latent variable models, many theoretical factors should be considered before applying those new techniques [67]. However, if subtypes of MDD do exist, using different statistical methods to reveal their structure could be worthwhile.

Thus, future analyses should ideally explore several advanced statistical techniques on enriched datasets. An investigation of the possibilities and limitations of different modeling techniques seems more reasonable than adhering exclusively to the latent factor and latent class models used up to now. Mega-analyses of the MDD symptoms of different samples could be worthwhile, as combined data may have a positive effect on robustness and generalizability of the results $[55,68]$. However, when performing mega-analyses, it is even more important to have rich datasets and to apply sophisticated modeling techniques to the data to accommodate interstudy heterogeneity [55]. Experiences with those new symptom-based classification attempts might inform other data-driven classification attempts that go beyond the DSM, such as the Research Domain Criteria [69,70].

Finally, what is the value of searching for data-driven subtypes of MDD? We started our review with the observation that patients with MDD differ considerably in their symptomatic presentation, with over 200 possible symptom combinations. To date, theoretical motivated subtypes have not resolved the substantial population heterogeneity of MDD. Empirical discernment of subtypes with similar symptoms could give an impetus to research on etiology, course, and treatment. Improved statistical tools are available to discover patterns in rich datasets, therefore, data-driven subtyping of depression is a valuable approach to be explored.

\section{Conclusions}

There is no conclusive evidence for the existence of depressive symptom dimensions or symptomatic subtypes in adults with MDD. Many theoretical and modeling choices affect the results of latent variable analyses. If there is any structure to be discovered in the current heterogeneity, consideration of those choices would provide an useful starting point for future studies.

\section{Additional material}

Additional file 1: Search strings. The search strings and results of the electronic database search.

Additional file 2: References of included studies. The references of the 20 studies included in this systematic review.

Additional file 3: Study characteristics. Study characteristics of the included latent class analyses (Table S1), and latent factor analyses (Table S2), including the translation of the original clusters identified by latent class analyses to a class ordering based on severity (Table S3).

\section{Abbreviations}

BDI: Beck Depression Inventory; BPRS: Brief Psychiatric Rating Scale; CA: cluster analysis; CFA: confirmatory factor analysis; DSM: Diagnostic and Statistical Manual of Mental Disorders; EFA: exploratory factor analysis; GMSAGECAT: Geriatric Mental State - Automated Geriatric Examination for Computer Assisted Taxonomy; HRSD: Hamilton Rating Scale for Depression; ICD: International Classification of Diseases; IDS (-C/-SR): Inventory for Depressive Symptomatology (Clinician/Self-Report); LCA: latent class analysis; MADRS: Montgomery and Åsberg Depression Rating Scale; MDD: major depressive disorder; PC: principal component; PCA: principal component analysis; RDC: Research Diagnostic Criteria; RMSEA: Root Mean Square Error of Approximation; SCL: Symptom Checklist; TLI: Tucker-Lewis Index; ZSDS: Zung Self-rating Depression Scale.

\section{Authors' contributions}

The study was designed by HMvL, PdJ and RAS. HMvL conducted the literature search. HMvL, PdJ and RAS assessed the eligibility of the studies for inclusion. HMvL extracted data from the included papers, and drafted the manuscript. JWR contributed to the interpretation of the results. PdJ, JWR, RCK, and RAS critically assessed drafts of the manuscript. All authors read and approved the final manuscript.

\section{Authors' information}

HMvL is a medical doctor and doctoral scholar at the University Center for Psychiatry (UCP), University Medical Center Groningen (UMCG), the Netherlands. PdJ is a professor at the UCP, UMCG and Center of Research on Psychology in Somatic Diseases at Tilburg University. JWR is professor at the Faculty of Philosophy of the University of Groningen. RCK is McNeil Family Professor of Health Care Policy at the Department of Health Care Policy at Harvard Medical School. RAS is professor and head of the UCP, UMCG.

\section{Competing interests}

The authors declare no competing interests.

\section{Acknowledgements}

PdJ was supported through a VICl grant (NWO grant 91812607;

Deconstructing Depression). JWR was supported through a VIDI grant (NWO grant 016114354; What are the chances?). RCK has had research support for his epidemiological studies from Analysis Group Inc., Bristol-Myers Squibb, Eli 
Lilly and Company, EPI-Q, GlaxoSmithKline, Johnson and Johnson Pharmaceuticals, Ortho-McNeil Janssen Scientific Affairs., Pfizer Inc., SanofiAventis Groupe, Shire US, Inc., and Walgreens Co.; has been a consultant for AstraZeneca, Analysis Group, Bristol-Myers Squibb, Cerner-Galt Associates, Eli Lilly and Company, GlaxoSmithKline Inc., HealthCore Inc., Health Dialog, Hoffman-LaRoche, Inc., Integrated Benefits Institute, John Snow Inc., Kaiser Permanente, Matria Inc., Mensante, Merck and Co, Inc., Ortho-McNeil Janssen Scientific Affairs, Pfizer Inc., Primary Care Network, Research Triangle Institute, Sanofi-Aventis Group, Shire US Inc., SRA International, Inc., Takeda Global Research and Development, Transcept Pharmaceuticals Inc., and WyethAyerst; has served on advisory boards for Appliance Computing II, Eli Lilly abd Company, Mindsite, Ortho-McNeil Janssen Scientific Affairs, Johnson and Johnson, Plus One Health Management and Wyeth-Ayerst; and owns a 25\% share in DataStat, Inc.

\section{Author details}

${ }^{1}$ Department of Psychiatry, University Medical Center Groningen, Hanzeplein 1, Groningen, 9713 GZ, The Netherlands. ${ }^{2}$ Faculty of Philosophy, University of Groningen, Oude Boteringestraat 52, Groningen, 9712 GL, The Netherlands. ${ }^{3}$ Department of Health Care Policy, Harvard Medical School, 180 Longwood Avenue, Boston, Massachusetts, 02115, USA.

Received: 5 July 2012 Accepted: 4 December 2012 Published: 4 December 2012

\section{References}

1. World Health Organization: The global burden of disease. 2004 update edition. Geneve: WHO; 2008.

2. Collins PY, Patel V, Joestl SS, March D, Insel TR, Daar AS, Scientific Advisory Board and the Executive Committee of the Grand Challenges on Global Mental Health, Anderson W, Dhansay MA, Phillips A, Shurin S, Walport M, Ewart W, Savill SJ, Bordin IA, Costello EJ, Durkin M, Fairburn C, Glass RI, Hall W, Huang Y, Hyman SE, Jamison K, Kaaya S, Kapur S, Kleinman A, Ogunniyi A, Otero-Ojeda A, Poo MM, Ravindranath V, Sahakian BJ, Saxena S, Singer PA, Stein DJ: Grand challenges in global mental health. Nature 2011, 475:27-30.

3. Belmaker RH, Agam G: Mechanisms of disease: major depressive disorder. N Engl J Med 2008, 358:55-68.

4. Cramer AO, Borsboom D, Aggen SH, Kendler KS: The pathoplasticity of dysphoric episodes: differential impact of stressful life events on the pattern of depressive symptom inter-correlations. Psychol Med 2012, 42:957-965.

5. Rhebergen D, Lamers F, Spijker J, de Graaf R, Beekman AT, Penninx BW: Course trajectories of unipolar depressive disorders identified by latent class growth analysis. Psychol Med 2012, 42:1383-1396.

6. Rush AJ: The varied clinical presentations of major depression disorder. J Clin Psychiatry 2007, 68:4-10.

7. Savitz JB, Drevets WC: Imaging phenotypes of major depressive disorder: genetic correlates. Neuroscience 2009, 164:300-330.

8. American Psychiatric Association: Diagnostic and Statistical Manual of Mental Disorders: DSM-IV-TR. Fourth, Text Revision edition. Arlington, US: American Psychiatric Publishing; 2000.

9. Feighner JP, Robins E, Guze SB, Woodruff RA Jr, Winokur G, Munoz R: Diagnostic criteria for use in psychiatric research. Arch Gen Psychiatry 1972, 26:57-63.

10. Spitzer RL, Endicott J, Robins E: Research diagnostic criteria: Rationale and reliability. Arch Gen Psychiatry 1978, 35:773-782.

11. Kendler KS, Munoz RA, Murphy G: The development of the Feighner criteria: a historical perspective. Am J Psychiatry 2010, 167:134-142.

12. Uher $R$, Muthén $B$, Souery $D$, Mors $O$, Jaracz J, Placentino A, Petrovic A, Zobel A, Henigsberg N, Rietschel M, Aitchison K, Farmer A, McGuffin P: Trajectories of change in depression severity during treatment with antidepressants. Psychol Med 2010, 40:1367-1377.

13. Elhai JD, Naifeh JA, Forbes D, Ractliffe KC, Tamburrino M: Heterogeneity in clinical presentations of posttraumatic stress disorder among medical patients: testing factor structure variation using factor mixture modeling. J Trauma Stress 2011, 24:435-443

14. Lubke $\mathrm{GH}$, Muthén B: Investigating Population Heterogeneity With Factor Mixture Models. Psychol Methods 2005, 10:21-39.

15. Stevens JP: Applied multivariate statistics for the social sciences. 5 edition. New York, NY US: Routledge/Taylor \& Francis Group; 2009.
16. Hutcheson GD, Sofroniou N: The multivariate social scientist: introductory statistics using generalized linear models London: SAGE; 1999.

17. James FC, McCulloch CE: Multivariate analysis in ecology and systematics: panacea or Pandora's box? Annual Review of Ecology and Systematics 1990, 21:129-166

18. de Jonge $P$, Mangano D, Whooley MA: Differential association of cognitive and somatic depressive symptoms with heart rate variability in patients with stable coronary heart disease: findings from the Heart and Soul Study. Psychosom Med 2007, 69:735-739.

19. Roest AM, Thombs BD, Grace SL, Stewart DE, Abbey SE, de Jonge P. Somatic/affective symptoms, but not cognitive/affective symptoms, of depression after acute coronary syndrome are associated with 12-month all-cause mortality. J Affect Disord 2011, 131:158-163.

20. American Psychiatric Association: Diagnostic and statistical manual of mental disorders: DSM-III. 3 edition. Washington, D.C.: American Psychiatric Association; 1980

21. American Psychiatric Association: Diagnostic and statistical manual of mental disorders: DSM-III-R. 3 edition. Cambridge: Press Syndicate of the University of Cambridge; 1987.

22. American Psychiatric Association: Diagnostic and statistical manual of mental disorders: DSM-IV. 4 edition. Arlington, VA US: American Psychiatric Publishing, Inc; 1994.

23. World Health Organization: International classification of diseases: manual of the international statistical classification of diseases, injuries, and causes of death: ICD-9. 9 edition. Geneva: WHO; 1977.

24. World Health Organization: International Statistical Classification of Diseases and related health problems: ICD-10. 10 edition. Geneva: WHO; 1994.

25. Copeland JR, Dewey ME, Griffiths-Jones H: A computerized psychiatric diagnostic system and case nomenclature for elderly subjects: GMS and AGECAT. Psychol Med 1986, 16:89-99.

26. Beck AT, Ward CH, Mendelson M, Mock J, Erbaugh J: An inventory for measuring depression. Arch Gen Psychiatry 1961, 4:561-571.

27. Derogatis LR: The Hopkins Symptom Checklist (HSCL): a self-report symptom inventory. Behav Sci 1974, 19:1-15.

28. Endicott J, Spitzer RL: A diagnostic interview: The schedule for affective disorders and schizophrenia. Arch Gen Psychiatry 1978, 35:837-844.

29. Hamilton M: A rating scale for depression. J Neurol Neurosurg Psychiatry $1960,23: 56-61$

30. Montgomery SA, Åsberg M: A new depression scale designed to be sensitive to change. Br J Psychiatry 1979, 134:382-389.

31. Overall JE, Gorham DR: The Brief Psychiatric Rating Scale. Psychol Rep 1962, 10:799-812.

32. Rush AJ, Giles DE, Schlesser MA, Fulton CL: The Inventory for Depressive Symptomatology (IDS): preliminary findings. Psychiatry Res 1986, 18:65-87.

33. Spitzer RL, Williams JB, Gibbon M, First MB: The Structured Clinical Interview for DSM-III-R (SCID): I. History, rationale, and description. Arch Gen Psychiatry 1992, 49:624-629.

34. Zung WW: A self-rating depression scale. Arch Gen Psychiatry 1965, 12:63-70.

35. Lux V, Aggen SH, Kendler KS: The DSM-IV definition of severity of major depression: inter-relationship and validity. Psychol Med 2010, 40:1691-1701.

36. Uher R, Farmer A, Maier W, Rietschel M, Hauser J, Marusic A, Mors O, Elkin A, Williamson RJ, Schmael C, Henigsberg N, Perez J, Mendlewicz J, Janzing JGE, Zobel A, Skibinska M, Kozel D, Stamp AS, Bajs M, Placentino A, Barreto M, McGuffin $\mathrm{P}$, Aitchison $\mathrm{KJ}$ : Measuring depression: Comparison and integration of three scales in the GENDEP study. Psychol Med 2008, 38:289-300.

37. Ohaeri JU, Otote DI: Family history, life events and the factorial structure of depression in a Nigerian sample of inpatients. Psychopathology 2002, 35:210-219.

38. Parker RD, Flint EP, Bosworth HB, Pieper CF, Steffens DC: A three-factor analytic model of the MADRS in geriatric depression. Int J Geriatr Psychiatry 2003, 18:73-77.

39. Romera I, Delgado-Cohen H, Perez T, Caballero L, Gilaberte I: Factor analysis of the Zung self-rating depression scale in a large sample of patients with major depressive disorder in primary care. BMC Psychiatry 2008, 8:4.

40. Fleck MPdA, Poirier-Littre M, Guelfi JD, Bourdel MC: Factorial structure of the 17-item Hamilton Depression Rating Scale. Acta Psychiatr Scand 1995, 92:168-172.

41. Steer RA, Beck AT, Riskind JH, Brown G: Relationships between the Beck Depression Inventory and the Hamilton Psychiatric Rating Scale for 
Depression in depressed outpatients. Journal of Psychopathology and Behavioral Assessment 1987, 9:327-339.

42. Haslam N, Beck AT: Categorization of major depression in an outpatient sample. J Nerv Ment Dis 1993, 181:725-731.

43. Galinowski A, Lehert P: Structural validity of MADRS during antidepressant treatment. Int Clin Psychopharmacol 1995, 10:157-161.

44. Corruble E, Legrand JM, Duret C, Charles G, Guelfi JD: IDS-C and IDS-SR: psychometric properties in depressed in-patients. J Affect Disord 1999, 56:95-101.

45. Maes M, Maes L, Schotte C, Cosyns P: A clinical and biological validation of the DSM-III melancholia diagnosis in men: results of pattern recognition methods. J Psychiatr Res 1992, 26:183-196.

46. Andreasen NC, Grove WM: The classification of depression: Traditional views versus mathematical approaches. Am J Psychiatry 1982, 139:45-52.

47. Schotte CKW, Maes M, Cluydts R, Cosyns P: Cluster analytic validation of the DSM melancholic depression. The threshold model: integration of quantitative and qualitative distinctions between unipolar depressive subtypes. Psychiatry Res 1997, 71:181-195.

48. Hybels CF, Blazer DG, Pieper CF, Landerman LR, Steffens DC: Profiles of depressive symptoms in older adults diagnosed with major depression Latent cluster analysis. Am J Geriatr Psychiatry 2009, 17:387-396.

49. Lamers F, De Jonge P, Nolen WA, Smit JH, Zitman FG, Beekman ATF, Penninx BWJH: Identifying depressive subtypes in a large cohort study: results from the Netherlands Study of Depression and Anxiety (NESDA) J Clin Psychiatry 2010, 71:1582-1589.

50. Parker G, Wilhelm K, Mitchell P, Roy K, Hadzi-Pavlovic D: Subtyping depression: testing algorithms and identification of a tiered model. J Nerv Ment Dis 1999, 187:610-617.

51. Davidson J, Woodbury MA, Pelton S, Krishnan KR: A study of depressive typologies using grade of membership analysis. Psychol Med 1988, 18:179-189.

52. Davidson JR, Woodbury MA, Zisook S, Giller EL: Classification of depression by grade of membership: a confirmation study. Psychol Med 1989, 19:987-998.

53. Grove WM, Andreasen NC, Young M, Endicott J: Isolation and characterization of a nuclear depressive syndrome. Psychol Med 1987, 17:471-484.

54. Gullion CM, Rush AJ: Toward a generalizable model of symptoms in major depressive disorder. Biol Psychiatry 1998, 44:959-972.

55. Major Depressive Disorder Working Group of the Psychiatric GWAS Consortium: A mega-analysis of genome-wide association studies for major depressive disorder. Mol Psychiatry 2012, doi: 10.1038/mp.2012.21.

56. MacCallum RC, Widaman KF, Zhang S, Hong S: Sample size in factor analysis. Psychol Methods 1999, 4:84-99.

57. Costello $A B$, Osborne JW: Best practices in exploratory factor analysis: four recommendations for getting the most from your analysis. Practical Assessment, Research \& Evaluation 2005, 10.

58. Schmitt TA: Current methodological considerations in exploratory and confirmatory factor analysis. Journal of Psychoeducational Assessment 2011, 29:304-321.

59. Fava M, Hwang I, Rush AJ, Sampson N, Walters EE, Kessler RC: The importance of irritability as a symptom of major depressive disorder: results from the National Comorbidity Survey Replication. Mol Psychiatry 2010, 15:856-867.

60. Kendler KS, Gardner CO: A longitudinal etiologic model for symptoms of anxiety and depression in women. Psychol Med 2011, 41:2035-2045.

61. Kessler RC, Gruber M, Hettema JM, Hwang I, Sampson N, Yonkers KA: Comorbid major depression and generalized anxiety disorders in the National Comorbidity Survey follow-up. Psychol Med 2008, 38:365-374.

62. Kessler RC, Petukhova M, Zaslavsky AM: The role of latent internalizing and externalizing predispositions in accounting for the development of comorbidity among common mental disorders. Current Opinion in Psychiatry 2011, 24:307-312.

63. Thompson B: Canonical correlation analysis: uses and interpretation Newbury Park: Sage; 1990.

64. Tay L, Diener E, Drasgow F, Vermunt JK: Multilevel mixed-measurement IRT analysis: An explication and application to self-reported emotions across the world. Organ Res Methods 2011, 14:177-207.

65. Lubke GH, Muthén B, Moilanen IK, McGough JJ, Loo SK, Swanson JM, Yang MH, Taanila A, Hurtig T, Järvelin M, Smalley SL: Subtypes versus severity differences in attention-deficit/hyperactivity disorder in the
Northern Finnish birth cohort. Journal of the American Academy of Child \& Adolescent Psychiatry 2007, 46:1584-1593.

66. Picardi A, Viroli C, Tarsitani L, Miglio R, de Girolamo G, Dell'acqua G, Biondi M: Heterogeneity and symptom structure of schizophrenia. Psychiatry Res 2012, doi:10.1016/j.psychres.2011.12.051.

67. Lubke G, Muthén BO: Performance of factor mixture models as a function of model size, covariate effects, and class-specific parameters. Struct Equ Modeling 2007, 14:26-47.

68. Cole DA, Cai L, Martin NC, Findling RL, Youngstrom EA, Garber J, Curry JF, Hyde JS, Essex MJ, Compas BE, Goodyer IM, Rohde P, Stark KD, Slattery MJ, Forehand R: Structure and measurement of depression in youths: Applying item response theory to clinical data. Psychol Assess 2011, 23:819-83.

69. Sanislow CA, Pine DS, Quinn KJ, Kozak MJ, Garvey MA, Heinssen RK, Wang PS, Cuthbert BN: Developing constructs for psychopathology research: Research domain criteria. J Abnorm Psychol 2010, 119:631-639.

70. Miller G: Psychiatry. Beyond DSM: seeking a brain-based classification of mental illness. Science 2010, 327:1437.

\section{Pre-publication history}

The pre-publication history for this paper can be accessed here: http://www.biomedcentral.com/1741-7015/10/156/prepub

doi:10.1186/1741-7015-10-156

Cite this article as: van Loo et al:: Data-driven subtypes of major

depressive disorder: a systematic review. BMC Medicine 2012 10:156.

\section{Submit your next manuscript to BioMed Central and take full advantage of:}

- Convenient online submission

- Thorough peer review

- No space constraints or color figure charges

- Immediate publication on acceptance

- Inclusion in PubMed, CAS, Scopus and Google Scholar

- Research which is freely available for redistribution

Submit your manuscript at www.biomedcentral.com/submit
Biomed Central 